\title{
Spots
}

\section{Neue Entwicklungen in der forensischen Musikpsychologie}

\author{
New Developments in Forensic Music Psychology
}

Klaus Frieler*a, Daniel Müllensiefen ${ }^{\mathrm{b}}$

[a] Unabhängiger Musikwissenschaftler und Musikgutachter, Hamburg, Deutschland. [b] Department of Psychology, Goldsmiths University of London, London, United Kingdom.

\section{Zusammenfassung}

Ausgehend von jüngsten Urheberrechtsurteilen, die die Musikwelt verunsichert haben, skizzieren wir in diesem Artikel, wie musikinformatische Methoden auf Basis musikpsychologischer Erkenntnisse eingesetzt werden könnten, um für mehr Rechtssicherheit der Musikschaffenden zu sorgen.

Schlüsse/wörter: Musikpsychologie, Urheberrecht, Plagiat, "Blurred lines”-Urteil, Musikinformatik, Kleine Münze

\begin{abstract}
Based on recent copyright rulings that have unsettled the music world, we outline in this article how music informatics methods based on music-psychological findings can be used to provide more legal certainty for music creators.

Keywords: music psychology, copyright infringement, plagiarism, "Blurred lines" ruling, music information retrieval, "Kleine Münze"

Jahrbuch Musikpsychologie, 2020, Vol. 29(1), Artikel e77, https://doi.org/10.5964/jbdgm.2019v29.77

Publiziert (VoR): 2020-10-22.

*Korrespondenzanschrift: Unabhängiger Musikwissenschaftler und Musikgutachter, Harkortstraße 162, 22765 Hamburg, Deutschland. E-Mail: kf@omniversum.de
(c) () ()
Dieser Open-Access-Artikel steht unter den Bedingungen einer Creative Commons Namensnennung 4.0 International Lizenz, CC BY 4.0 (https://creativecommons.org/licenses/by/4.0/deed.de). Diese erlaubt für beliebige Zwecke (auch kommerzielle) den Artikel zu verbreiten, in jedwedem Medium zu vervielfältigen, Abwandlungen und Bearbeitungen anzufertigen, unter der Voraussetzung, dass der Originalartikel angemessen zitiert wird.

Als am 21.03.2018 der Court of Appeals for the Ninth Circuit das finale Urteil im Fall Willams vs. Gaye verkündete, war die internationale Musikszene zutiefst verunsichert. Was war passiert? Das US-amerikanische Berufungsgericht hatte das Urteil eines kalifornischen Bezirksgerichts bestätigt und den Erben Marvin Gayes 5 Mio. Dollar Schadensersatz zugesprochen, weil der Megahit „Blurred Lines“ von Robin Thicke und Pharell Williams aus dem Jahr 2013 gegen das Urheberrecht des Marvin-Gaye-Klassikers „Got to give it up“ (1977) verstoßen haben soll. Das Urteil wurde vielfach als Paradigmenwechsel in der musikalischen Urheberrechtsprechung wahrgenommen, da zum ersten Mal nicht melodische Übernahmen zentral für die Entscheidung waren, sondern der Gesamteindruck und die Spezifika des Grooves der beiden Stücke. Stilistische Merkmale galten bis dato als nicht schutzfähig, sondern als musikalisches Allgemeingut oder Handwerk. Diese Rechtspraxis ließ viel Spielraum für musikalische Inspirationen. Solange keine Melodien oder Texte betroffen waren, waren Übernahmen rechtlich relativ ungefährlich. Doch diese Praxis steht seit dem „Blurred Lines“-Urteil fundamental in Frage und sorgt damit für Rechtsunsicherheiten bei Musikschaffenden, gerade in der aktuellen Popmusik, 
die so stark wie nie zuvor auf der direkten Weiterverarbeitung fremden Materials beruht. Die technischen Möglichkeiten moderner digitaler Audioworkstations und die einfache Verfügbarkeit von Samplelibraries und fertigen Loops erlauben nicht nur, fremdes Material zu übernehmen und zu verfremden, sondern sie fordern geradezu dazu heraus. Zudem bedient sich die Produktion moderner Popmusikwerke immer öfter industrieller Produktionsmethoden wie Arbeitsteilung und die Verwendung vorgefertigte Bauteile wie Loops und Grooves (Seabrook, 2015)i. Diese Logik der Hitproduktion führt in einer positiv-rückgekoppelten Schleife zur Evolution erprobter musikalischer Tropen, d.h. zu Melodieformeln, Akkordklischees und gängigen Formschemata, die das Hitpotential steigern sollen. Songs sollen zwar neu, kreativ und originell sein, aber gleichzeitig Bedürfnisse der Hörer:innen nach Altbekanntem bedienen, denn diese lieben - überspitzt formuliert - in der Regel nur was sie bereits kennen, was auch auf grundlegenden Wahrnehmungs- und Verarbeitungsmechanismen wie dem mere exposure effect (Zajonc, 1968), der availability heuristic (Tversky \& Kahneman, 1973) oder dem chunking (Miller, 1956) beruht. Eine Lösung für die Spannung zwischen Originalität und Bekanntheit ist die Rekombination von Elementen aus einem großen Repertoire popmusikalischer Bausteine. Das Baukastensystem für musikalische Primärmerkmale wie Rhythmik, Harmonik und Melodik ist in der kommerziellen Popmusik relativ stark kodifiziert (Frieler \& Müllensiefen, 2020b), wird aber vor allem durch Innovationen im Produktionsbereich kontrastiert, also Instrumentierung (Sound) und Arrangement, die stark von digitalen Produktionsmitteln getrieben werden. Wie aber der Fall „Blurred Lines“ zeigt, orientiert sich auch diese Produktionsebene bewusst oder unbewusst oft an bekannten Vorbildern, was bisher problemlos möglich war und was nun in Frage gestellt ist.

Das Urheberrecht soll auf der einen Seite Urheber:innen und deren Werke schützen, damit für eine angemessene Vergütung sorgen und so Anreize für kreative Tätigkeiten schaffen. Auf der anderen Seite soll es auch den kreativen Austausch von Ideen und einen lebendigen musikalischen Diskurs ermöglichen. Dieser Ausgleich ist jedoch schwierig, weil die Grenze zwischen musikalischem Allgemeingut und Handwerk einerseits und schutzfähiger kreativer Schöpfung andererseits unscharf ist, besonders da durch die Praxis der „Kleinen Münze“ (Schulze, 1983) die Anforderungen an die Schutzfähigkeit sehr niedrig angesetzt sind. Das führt zu einer ständigen latenten Bedrohung für Musikschaffenden, im Falle eines großen Hits auf Urheberrechtsverletzung verklagt zu werden, wobei kaum allgemein verbindliche Kriterien erkennbar sind, da letztlich immer Fall-basiert entschieden wird.

In einem kürzlich erschienenen Aufsatz (Frieler \& Müllensiefen, 2020c) haben wir argumentiert, dass das Hauptproblem darin besteht, dass bereits kurze Teile eines längeren Werks durch die Kleine Münze Schutz genießen. Dabei haben wir anhand einer Korpusstudie mit Popsongmelodien gezeigt, dass diese zur Gänze aus kurzen Melodieschnipseln bestehen, die in vielen anderen Songs vorkommen. Dies gilt insbesondere, wenn man nur Tonhöhenintervalle betrachtet und die rhythmische Dimension ausblendet.

Aufbauend auf den Ergebnissen von Frieler und Riedemann (2011), wo gezeigt wurde, dass auch Laien in der Lage sind, in kurzer Zeit zu einem vorgegebenen Backingtrack Melodien zu schaffen, wäre eine Lösung der urheberrechtlichen Probleme die Komposition von Melodien als musikalisches Handwerk anzusehen. Melodiekomposition als musikalisches Handwerk zu betrachten käme allerdings faktisch einer Abschaffung von §24, 2 („starrer Melodienschutz“) des deutschen Urhebergesetzes gleich, mit weitreichenden Konsequenzen für die Urheberrechtspraxis. 
Ein anderer Lösungsansatz, der durch aktuelle Entwicklungen in der Musikinformatik in Reichweite gelangt ist, ist eine objektive Einschätzung der Schutzfähigkeit streitgegenständlicher Melodien. Wie Frieler und Müllensiefen (2020a) gezeigt haben, könnte man die Individualität und damit deren Schöpfungshöhe einer Popmelodie anhand des mittleren Informationsgehalts ihrer melodischen Bestandteile in Bezug auf einen Korpus von Melodien abschätzen. Je häufiger die Bestandteile im Korpus auftauchen, desto geringer ist deren Informationsgehalt und als desto geläufiger wird die Melodie wahrgenommen. Diese Schätzung kann dann als richtungsweisende Information in Urheberrechtsstreitigkeiten dienen.

Des Weiteren bieten Vergleichsdatenbanken generell die Möglichkeit, die Gewöhnlichkeit oder Stilkonformität eines Songs quantitativ einzuordnen oder mithilfe von psychologisch validierten Ähnlichkeitsmaßen (Cason \& Müllensiefen, 2012; Müllensiefen \& Frieler, 2004; Müllensiefen \& Pendzich, 2009) weitere Melodien zu finden, die den streitgegenständlichen Melodien ähneln und so eine Einordnung eines Falles auf qualitative Weise ermöglichen. Dies kann auch auf Stil- und Klangqualitäten ganzer Tracks mit Hilfe entsprechender Datenbanken und Algorithmen ausgeweitet werden.

In jedem Fall wird deutlich, wie musikpsychologische Forschung einen Beitrag zur Diskussion um die Novellierung des Urheberrechts und auch zur juristischen Praxis beitragen kann. Moderne musikinformatische Verfahren, die auf diesen Forschungsergebnissen basieren, haben ein großes Potenzial, für mehr Rechtssicherheit und klarere Kriterien in Auseinandersetzungen zu sorgen. Allerdings muss die musikpsychologische Basis dieser Verfahren noch systematisch durch robuste empirische Studien weiter ausgebaut werden.

\section{Anmerkungen}

i) Ob dies in der Tat neuer Trend ist, muss aber dahingestellt bleiben, denn Hitfabriken wie Tin Pan Alley, das Brill Building, Motown, Stock-Aitken-Waterman, Max Martin, oder in Deutschland Frank Farian, Ralph Siegel und Dieter Bohlen, zeigen, dass Massenproduktionstechniken seit langem sehr erfolgreich in der Popmusik funktionieren.

\section{Finanzierung}

Die Autoren/die Autorinnen haben keine Finanzierung für das Forschungsprojekt erhalten.

\section{Interessenkonflikte}

Die Autoren/Autorinnen haben erklärt, dass keinerlei kunkurrierende Interessen bestehen.

\section{Danksagung}

Die Autoren/die Autorinnen haben keine Unterstützung erhalten.

\section{Literatur}

Cason, R. J. S. \& Müllensiefen, D. (2012). Singing from the same sheet: Computational melodic similarity measurement and copyright law. International Review of Law Computers \& Technology, 26(1), 25-36. https://doi.org/10.1080/13600869.2012.646786

Frieler, K. \& Müllensiefen, D. (2020a, März 6-7). Kleine Münze, große Fragen. Musikalische Schöpfungshöhe aus der Perspektive empirischer Musikforschung [Vortrag]. Tagung der Fachgruppe „Systematischer Musikwissenschaft“ der Gesellschaft für Musikforschung, Kassel, Deutschland. 
Frieler, K., \& Müllensiefen, D. (2020b). Song and harmonic templates in popular music. In P. Tillessen \& T. Prill (Hrsg.), Whatever (pp. 97-103). Leipzig, Deutschland: Spector Books.

Frieler, K., \& Müllensiefen, D. (2020c, Februar 20-21). Kleine Münze, große Fragen. Musikalische Schöpfungshöhe aus der Perspektive empirischer Musikforschung. In K. Hondros, S. Beaucamp, G. Fischer \& S. Schroer (Hrsg.), Tipping Points. Zum Verhältnis von Freiheit und Restriktion im Urheberrecht [Tagung]. Die interdisziplinäre Fachtagung des Weizenbaum-Instituts für die vernetzte Gesellschaft und des Fachausschusses Urheberrecht der Gesellschaft für Musikwirtschafts- und Musikkulturforschung (GMM), Berlin, Deutschland.

Frieler, K. \& Riedemann, F. (2011). Is independent creation likely to happen in pop music? Musicae Scientiae, 15(1), 17-28. https://doi.org/10.1177/1029864910393406

Miller, G. A. (1956). The magical number seven, plus minus two: Some limits on our capacity for processing information. Psychological Review, 63(2), 81-97. https://doi.org/10.1037/h0043158

Müllensiefen, D. \& Frieler, K. (2004). Cognitive adequacy in the measurement of melodic similarity: Algorithmic vs. human judgments. Computing in Musicology, 13, 147-176.

Müllensiefen, D. \& Pendzich, M. (2009). Court decisions on music plagiarism and the predictive value of similarity algorithms. Musicae Scientiae, 13(1_suppl), 257-295. https://doi.org/10.1177/102986490901300111

Schulze, G. (1983). Die kleine Münze und ihre Abgrenzungsproblematik bei den Werkarten des Urheberrechts. Zürich, Schweiz: HochschulVerlag.

Seabrook, J. (2015). Song machine: Inside the hit factory. New York, NY, USA: Norton \& Company.

Tversky, A. \& Kahneman, D. (1973). Availability: A heuristic for judging frequency and probability. Cognitive Psychology, 5(2), 207-232. https://doi.org/10.1016/0010-0285(73)90033-9

Zajonc, R. B. (1968). Attitudinal effects of mere exposure. Journal of Personality and Social Psychology, 9(2, Pt.2), 1-27. https://doi.org/10.1037/h0025848 